\title{
La conciencia fonológica en contextos educativos y terapéuticos: efectos sobre el aprendizaje de la lectura
}

Luz Viviana Sastre-Gómez

Institución Universitaria Politécnico Grancolombiano, Colombia lvsatsre@poligran.edu.co orcid.org/00oo-0002-1748-6436

\section{Juan David Roa-De la Torre}

Institución Universitaria Politécnico Grancolombiano, Colombia juandavidroa@gmail.com orcid.org/0000-0002-4158-057X
Nancy Mireya Celis-Leal

Institución Universitaria Politécnico Grancolombiano, Colombia mireyacel@gmail.com orcid.org/0ooo-0003-1845-7183

Christian Felipe Luengas-Monroy

Institución Universitaria Politécnico Grancolombiano, Colombia fluengas@poligran.edu.co orcid.org/0000-0001-8145-1230

\section{Resumen}

El aprendizaje de la lectura supone el desarrollo previo de una serie de habilidades, que van permitiendo a los niños la construcción y ganancia de destrezas en diferentes dimensiones hacia la consecución del progreso de sus procesos cognitivos. Entre ellas, se encuentra la conciencia fonológica, variable de mayor relevancia en cuanto al desarrollo lector se refiere. La eficiencia de dicho mecanismo se relaciona directamente con el éxito en la alfabetización; las dificultades tanto para aprender a leer como para la lectura en sí misma, históricamente se han asociado a un déficit en el componente fonológico del lenguaje. El presente artículo evidencia las distintas maneras en que, tanto el contexto pedagógico como el terapéutico, se han generado estrategias facilitadoras que influyen en el normal desarrollo de las habilidades pre lectoras y de adquisición de la lectoescritura.

\section{Palabras clave}

Conciencia fonológica; enseñanza de la lectura y la escritura; educación escolar; educación especial; terapia del lenguaje (Fuente: Tesauro de la Unesco). 


\title{
Phonological Awareness in Educational and Therapeutic Contexts: Effects on Learning to Read
}

\begin{abstract}
Learning to read implies the prior development of a series of skills that allow children to build and gain abilities in different dimensions that contribute towards progress in their cognitive processes. One of these skills is phonological awareness, which is a particularly relevant variable as far as the reader's development is concerned. The efficiency of this mechanism is related directly to success in becoming literate. The difficulties in learning to read, and in reading itself, have been associated historically with a deficit in the phonological component of language. This article shows the different ways in which educational and therapeutic contexts have helped to generate facilitating strategies that influence the normal development of pre-reading skills and those for becoming literate.
\end{abstract}

Keywords

Phonological awareness; teaching reading and writing; school education; special education; speech therapy (Source: Unesco Thesaurus). 


\section{A consciência fonológica em contextos educativos e terapêuticos: efeitos sobre a aprendizagem da leitura}

Resumo

A aprendizagem da leitura representa o desenvolvimento prévio de uma série de habilidades, permitindo que as crianças construam e tirem proveito de capacidades em diferentes dimensões rumo ao progresso dos seus processos cognitivos. Entre elas, encontra-se a consciência fonológica, variável de maior relevância referente ao desenvolvimento leitor. A eficiência desse mecanismo se relaciona diretamente com o sucesso na alfabetização; as dificuldades tanto para aprender a ler como para a leitura em si têm-se associado historicamente a um déficit no componente fonológico da linguagem. O presente artigo evidencia as diferentes maneiras em que, tanto no contexto pedagógico quanto no terapêutico, foram criadas estratégias facilitadoras que influem no desenvolvimento normal das habilidades pré-leitoras e de aquisição da alfabetização.

\section{Palavras-chave}

Consciência fonológica; ensino de leitura e escrita; educação escolar; educação especial; terapia da linguagem (Fonte: Tesauro da Unesco). 


\section{Revisión teórica}

Aprender a leer se constituye en un importante reto para los escolares, en cuanto no solo requiere un despliegue de habilidades por sí mismo, sino que supone la suma de diversos factores que pueden determinar el futuro de los nuevos lectores. Según Márquez y De la Osa (2003, p. 358), "se traduce en resultados personales, sociales y económicos significativos en nuestra cultura". El aprendizaje de la lectura depende de variables tan diversas y complejas como las que están dadas por el contexto cultural, la madurez y, por supuesto, las particularidades de la escuela (Arancibia, Bizama y Sáez, 2012). Es en esta última donde los niños se enfrentan directamente con tal avezada labor, al pasar de la oralidad y la construcción expresiva del lenguaje a una primera fase de decodificación y codificación de signos y símbolos y remontar la arbitrariedad de estos y consturir significados (Aguilar et al., 2011).

De acuerdo con lo que proponen Solsona, $\mathrm{Na}$ varro y Aguilar (2005), la escuela deviene el nicho favorecedor y potenciador de los procesos cognitivos del niño, y es, sin duda, el desarrollo lector y la adquisición de la lengua escrita los que determinan un periodo crítico, ya que implican variables de aprendizaje personal, métodos propios de cada institución y exposición, en muchos casos, a un segundo idioma. Bravo (2002) sostiene que aquel "nicho" tiene lugar indiscutiblemente en la escuela, donde el papel del maestro es fundamental en el establecimiento de relaciones y asociaciones; en otras palabras, en la construcción dinámica del aprendizaje inicial de la lectura que entiende

la conciencia fonológica como una zona de desarrollo próximo (ZDP), que implica para el niño que está en condiciones de aprender a leer, dos componentes centrales: el nivel de conciencia fonológica oral, y la interacción de los componentes escuchados de las palabras con el lenguaje escrito. (pp. 166-167)
El desarrollo del proceso lector y sus posibles dificultades se relaciona directamente con la conciencia fonológica, definida por Bravo (2006, p. 53) como "la toma de conciencia de los componentes fonéticos del lenguaje oral y el dominio de diversos procesos que los niños pueden efectuar conscientemente sobre el lenguaje oral". Además, se constituye como el predictor más importante en el desarrollo de las competencias lectoras, ya que va desde la sensibilidad fonológica hasta la conciencia propiamente dicha, entendida esta última como un proceso metalingüístico de acuerdo con Rosselli, Matute y Ardila (2010).

Por su parte, para Núñez y Santamarina (2014), la conciencia fonológica es definida como

la capacidad de reflexionar sobre los elementos fonológicos estructurales, componentes formales del lenguaje oral y manipularlos, por ello, incluye la habilidad para operar con los segmentos de las palabras, es decir, segmentar las unidades más pequeñas, tales como sílabas, sonidos, fonemas y unidades intrasilábicas (análisis fonológico), al mismo tiempo que se posee la habilidad para crear nuevas unidades superiores a partir de dichos segmentos más pequeños aislados (sintesis fonológica). (p. 86)

El entendimiento y abordaje de esta magnífica habilidad ha motivado su estudio desde diferentes posturas epistemológicas; no obstante, todas coinciden en su importancia. Desde la escuela, el desafío incluye, en primera instancia, el desarrollo del nivel fonológico de los niños, pues de este dependerá, según Márquez y De la Osa Fuentes (2003), el conocimiento y el éxito que tendrán más adelante en labores relacionadas con el reconocimiento, la identificación y la diferenciación de los fonemas y de las reglas de la lengua que los gobierna en sus posibles combinaciones, para formar sílabas y palabras.

Esta postura es compartida por Jamet (2006), quien considera fundamental tener en cuenta el grado de sensibilidad intrasilábico que presentan 
los escolares a los 6 años y medio, puesto que podría constituirse como predictor de la habilidad fonémica, que además es fundamental durante el proceso del aprendizaje de la lectura e influir en el crecimiento de las habilidades aritméticas, como lo proponen Solsona, Navarro y Aguilar (2005), con un debido entrenamiento, y como lo relacionan Michalczyk, Krajewski, Prebler y Hasselhorm (2013) en tareas específicas concernientes con los componentes de la memoria de trabajo.

La caracterización de la conciencia fonológica incluye el "desarrollo de habilidades tales como: detección de rimas, identificación de sílabas, igualación de sonidos iniciales y conteo de fonemas, comparación de la longitud de las palabras y representación de los fonemas con letras" (Hoien, Lundberg, Stanovich y Bjaalid, 1995, citado en Bravo, 2002). Así es como los escolares se enfrentan a estas tareas e inician el desarrollo de habilidades para comparar y "manipular" estas unidades y descubren que forman en conjunto palabras y más adelante reconocen las sílabas. Sin embargo, se debe considerar como aspecto fundamental la transparencia de la lengua materna, como en el caso del español y el italiano, y en contraposición la opacidad del inglés y el francés, más teniendo en cuenta si el tipo de educación es bilingüe (Anthony y Francis, 2005), si el modelo educativo considera el inicio temprano o no de la alfabetización y si otorga más valor a las tareas de lectura o escritura.

Entonces, se puede entender la conciencia fonológica como aquella conciencia que llega a tener cada persona sobre su propia lengua que discrimina reflexivamente los patrones de correspondencia entre letra y sonido, los cuales están implícitos en el proceso de lectura y escritura, que, además, incluyen el análisis grafémico, la asignación y el ensamblaje de fonemas, como lo describe Cuetos (2011).

Las investigaciones efectuadas en distintos idiomas desde la primera infancia hasta la primaria confirman que la conciencia fonológica se convierte en la base para el aprendizaje de la lectura en todos los idiomas, como se detalla a continuación:

Estudios en Inglés, (Compton, 2000; O'Connor y Jenkins, 1999, Stanovich, 2000), en Holandés (De Jong y Van der Leij, 1999) en Portugués, (Cardoso, Martins, 2001); en Español (Bravo y Orellana, 1999; Bravo, Villalón y Orellana, 2000; Carrillo 1994; Carrillo y Marín 1996; Defior 1996); y en Francés (Sprenger-Charolles, Siegel y Bonnet, 1998). (Bravo, 2002, pp. 173-175)

Esto confirma aún más la necesidad de desarrollar procesos cognitivos fundamentales para interactuar con la enseñanza de la lectura y considerar las variables de cada lengua, como pasa en inglés con las unidades intrasilábicas que muestran mayor estabilidad en la correspondencia entre las formas escritas y habladas, por ejemplo en las rimas; o en el caso del español, donde son más frecuentes las palabras polisílabas con límites fonológicos claros entre las sílabas.

Así podríamos esperar que la utilidad de la conciencia fonológica intrasilábica, frente a la conciencia fonológica silábica, no sea idéntica a la que en este nivel puede tener en la lengua inglesa (Márquez y De la Osa, 2003). Por esto, en nuestra lengua, se pueden presentar variables que interfieren directamente en la adquisición de la conciencia fonológica, ya que la lectura es un sistema alfabético, el cual requiere de un proceso de codificación de la información verbal en la memoria, de modo que se logren mantener los fonemas que representan las letras, las partes de las palabras y las palabras ya procesadas. (Baddeley, 2012)

También se han encontrado evidencias de deficiencias en el aprendizaje de la correspondencia fonema-grafema y dificultades en el acceso fonológico en personas con otras lenguas, como en el cantonés (Su et al., 2011).

En los primeros años escolares, se debe iniciar el entrenamiento en percepción visual y auditiva, 
comprensión y producción oral, atención y motricidad, de esta manera se favorece el proceso lector, puesto que se estimula la comprensión oral, la discriminación auditiva y el nivel cognitivo y permite que se organicen las estructuras para el aprendizaje (memoria operativa y semántica) y se facilite la adquisición de la lectura y la escritura. Como lo anotan Herrera y Defior (2005), la segmentación de las unidades del habla y su ensamblaje con los grafemas es uno de los problemas fundamentales que enfrentan los niños prelectores, por lo que alcanzar la suficiencia en dichas habilidades implica para los preescolares "comprender que existe una relación entre los fonemas y los grafemas" (Cuadrado y Trías, 2008, p. 2), y poder realizar otras labores, como las descritas por dichos autores, quienes además anotan que el niño "debe ser capaz de reconocer los diferentes sonidos, separarlos, asociarlos y descubrir que, distintas combinaciones pueden conformar palabras diferentes" (p. 2). Si se usan estas difíciles labores en un contexto bilingüe, el papel de la conciencia fonológica es fundamental, ya que, según Éva y Réka (2013), se presenta una relación directamente proporcional entre la conciencia fonológica y la adquisición de un segundo idioma, que si bien está mediada por el nivel de desarrollo de habilidades, se trata, sin lugar a duda, de un proceso transferencial, de la lengua materna a la segunda lengua, que presenta un valor predictivo importante en el rendimiento lector de los escolares.

Asimismo, las variables mencionadas son tenidas en cuenta dentro de contextos escolares que realmente buscan favorecer los procesos de adquisición de la conciencia fonológica, por lo que iqué puede pasar en ambientes escolares donde prima la vulnerabilidad en el ámbito familiar, social y educativo? "Todavía existen fuertes diferencias de rendimiento entre escuelas de dependencia privada y de dependencia pública, desigualdades que se traducirán en un futuro próximo en diferencias de oportunidades para los escolares de sectores más vulnerables de la población" (Bizama et al., 2013). Para que un contexto escolar sea apropiado, debe contar con interacciones entre las habilidades cognitivas y psicolingüísticas de los niños, con otros factores como libros o textos a los que tenga fácil acceso, con estrategias metodológicas y didácticas, proporcionadas por los docentes, y con un ambiente familiar igualmente estimulado, sin desconocer otras variables como el desarrollo del sustrato neurobiológico.

\section{Memoria de trabajo, conciencia fonológica y sus posibles dificultades}

La memoria se constituye por sí misma en una de las habilidades cognitivas por excelencia en el ser humano, dado que es gracias a ella que logramos ser y hacer en cuanto nos permite representarnos el mundo. De acuerdo con Ardila y Ostrosky (2012), esta desempeña un papel fundamental en el desarrollo de todos los aspectos de nuestra vida, es una función de alta complejidad, posibilita la adaptación e interacción contextual, pues dependemos de los recuerdos para estructurar las vivencias, generar respuestas y aprender. Según Rosselli, Matute y Ardila (2010), la adquisicón de diversidad de habilidades congnitivas se media necesariamente por la memoria. Sin lugar a duda, ésta última está estrechamente ligada con la conciencia fonológica.

La memoria corresponde directamente con el proceso de aprendizaje, más cuando es entendida como un sistema dinámico. Tal y como lo explica Coll (2011, p. 11), "la manera de aprender puede variar en función de la tarea que estemos realizando, así como de otros factores", entre ellos las formas variadas de almacenamiento, que se relacionan con los diversos sistemas cerebrales de memorizar y que tienen que ver directamente con las etapas del proceso temporal de la memoria.

El abordaje de la memoria de trabajo es determinante, dado que se relaciona directamente con el proceso de aprendizaje y posteriormente con el desarrollo de habilidades escolares; la memoria operativa, de acuerdo con lo descrito por Rosselli, Matute 
y Ardila (2010), va madurando en la medida en que el niño va pudiendo controlar su conducta que logra "inhibir proactiva y retroactivamente" entre los 6 y 8 años y alcanza su máximo desarrollo hacia los 12 años. El término de memoria de trabajo se ha ido robusteciendo y consolidando en los últimos años. Una definición más amplia es dada por Tirapu-Ustárroz y Muñoz-Céspedes (2005, p. 476): "Se define como un sistema que mantiene y manipula la información de una manera temporal, por lo que interviene en importantes procesos cognitivos, como la comprensión del lenguaje, de la lectura y de diversas formas de razonamiento". Asimismo, dichos autores explican que actúa como un sistema que provee el almacenamiento temporal de la información y permite el aprendizaje de nuevas tareas. Si se logra tener la información almacenada por cierto periodo y operar con ella, transformarla en nuevos constructos, entonces se ejecuta una labor de alto nivel, puesto que posibilita la relación de información y el logro de objetivos inmediatos, como lo plantea Baddeley (2003), tras lo cual se alcanza la resolución de problemas, en una interacción dinámica.

Según Baddeley (2003), la memoria de trabajo es un sistema de capacidad limitada, que además de mantener la información, la almacena. También, le permite a la persona mejores procesos de pensamiento, proporcionando una interfaz entre la memoria a largo plazo y la percepción. Por su parte, Baddeley (2012) propone una "división" o un sistema tripartito que incluye el ejecutivo central, el bucle fonológico y la agenda visoespacial; los dos últimos actúan como suplementarios del ejecutivo central, el cual es definido como el componente más complejo e importante de la memoria operativa, pues asume la labor de coordinador de recursos, capaz de enfocar la atención, almacenar y tomar decisiones; subordinados periféricos de este, encontramos el bucle articulatorio o fonológico, responsable de la información relacionada con el habla y la agenda visoespacial, cuya labor tiene que ver con la organización y manipulación de la información de este tipo.
El bucle fonológico, detallado por Baddeley (2003), comprende el almacén fonológico, que guarda información acústica por 1 o 2 segundos y el proceso de control articulatorio, referido al habla subvocal. Sus funciones incluyen mantener la información dentro del almacén y permitir el trabajo con dicho material. En un sentido más amplio, se puede atribuir la función de "soporte" o respaldo del habla, en caso de ser necesario, ya que es importante para nuevos aprendizajes fonológicos a largo plazo. Los hallazgos referidos al bucle fonológico encierran una serie de efectos, como lo son el efecto de similitud acústica, en el cual al tener dos unidades muy parecidas es más difícil su recordación; el efecto del habla irrelevante, donde se simplifica el accionar del recuerdo por la relevancia de este, no siendo notables ni la atención ni la semántica; el efecto de la longitud de la palabra, donde la recordación está mediada por la longitud de la palabra y se relaciona directamente con el spam de memoria, ya que son inversamente proporcionales; y el efecto de supresión articulatoria, el cual previene que se repita lo que se trata de recordar y también evita que se guarden estímulos visuales en el almacén fonológico. En suma, el bucle fonológico, relacionado con la percepción y la producción del habla, la agenda visoespacial y su correlación con los procesos de percepción visual-acción y el ejecutivo central, que comanda los dos anteriores y es el regulador de la atención y el comportamiento, conforma la triada más efectiva y probablemente más evolucionada que facilita la interfaz entre la memoria a corto y largo plazo,y, por ende, esencial en el proceso de aprendizaje, como lo explican en Arancibia et al. (2012), con respecto a la correlación positiva que encontraron entre la memoria verbal y la segmentación fonológica, donde queda de manifiesto que el desarrollo de esta habilidad es directamente proporcional a la capacidad de manipular los elementos verbales y la habilidad para poder acceder a la lengua escrita.

De acuerdo con Zayed, Roehrig, Arrastia-Lloyd y Gilgil (2013), la conciencia fonológica y la memoria de trabajo guardan una estrecha relación, además 
de considerarse como indicadores importantes por tener en cuenta en el aprendizaje de la lectura, ya que cualquier alteración sutil puede tener efecto en el desarrollo lector, incluso en niños con lengua diferente del español, como es el caso de preescolares egipcios, cuya lengua materna es el árabe. Los estudios sugieren una directa relación entre conciencia fonológica, memoria de trabajo verbal y el riesgo de dislexia. Cuando se logran detectar factores de riesgo, como los relacionados con las fallas en el procesamiento fonológico y en el rendimiento en la memoria operativa verbal, se pueden generar estrategias para mejorar el procesamiento de información.

Según Vargas y Villamil (2007), los procesos de lectura y escritura son definitivamente complejos. Lógicamente, se adquieren con posterioridad al desarrollo del habla, lo cual hace que las habilidades implicadas sean altamente especializadas y refinadas (decodificación, codificación, organización y creatividad), postura reafirmada por Herrera y Defior (2005), donde se explica la transición de la oralidad a la escritura y se resalta el papel de la conciencia fonológica como aspecto decisivo en el éxito de la lectoescritura; este periodo se constituye en el primer momento de observación importante en cuanto al desarrollo metalingüístico, pues es precisamente esta etapa la que puede definir el desarrollo y consolidación del proceso lector escolar.

De acuerdo con Preston, Hull y Edwards (2013), en los preescolares resulta un poco más sencillo atender a indicadores o predictores del desarrollo de la conciencia fonológica, como es el caso de los niños que presentan errores en la producción de los sonidos del habla (procesos fonológicos), puesto que posteriormente pueden presentar un trastorno articulatorio (funcional, específicamente), que repercuta en la ganancia de habilidades fonológicas, pues típicamente obtienen puntuaciones bajas en tareas de procesamiento fonético-fonológico y algunos casos derivan en trastornos de lectura. Es decir que las dificultades en el aula que devienen errores pueden explicitarse como ciertas dificultades en la manera en como se presenta la representación fonológica superior, pues las habilidades en conciencia fonológica se relacionan directamente con la calidad de dichas representaciones fonológicas subyacentes y se consideran un aspecto significativo en tareas de decodificación de lectura y ortografía.

En un sentido más amplio, dicha habilidad metalingüística se constituye en sí misma como "el mejor predictor" de éxito en labores de lectura y escritura, incluso cuando se trata de un trastorno específico del lenguaje, especialmente expresivo, según lo relacionado por Coloma, Cárdenas y De Barberi (2005), en los estudios realizados con preescolares. En ellos, se analizó la correlación entre el trastorno específico del lenguaje, las fallas en la conciencia fonológica y la escritura que determinó que un alto porcentaje de niños (del estudio) con trastorno específico del lenguaje presenta dificultades fonológicas o escriturales que los constituye en un grupo de riesgo. Sin embargo, no se puede generalizar como síntoma causal, aunque, por otro lado, algunas evidencias logran documentar el efecto de las dificultades de aprendizaje en tareas relacionadas con la conciencia fonológica (segmentación fonológica), la comprensión verbal y habilidades narrativas, como lo describen Gómez et al. (2007).

La dificultad de la lectura más conocida es la dislexia. Según el Ministerio de Educación, Cultura y Deporte Español (2012), la dislexia es un trastorno neurobiológico, "es decir, existe una alteración que afecta a la funcionalidad de la conducta lectora que impide al sujeto extraer correcta y eficazmente la información escrita y, por tanto, afecta a su adaptación académica, personal y social" (p. 26), es específico e independiente del nivel de inteligencia, excluye déficits sensoriales, emocionales, contextuales y lesiones neurológicas sobrevenidas. De acuerdo con la descripción y el análisis de Artigas-Pallarés (2009), la dislexia tiene una vinculación con los genes cuantitativos y pleiotrópicos, no de un solo gen sino de varios, comparte algunas características como la comorbilidad con otros trastornos, la alta influencia 
del entorno, la diferenciación cualitativa de los síntomas y la imposibilidad de determinar un marcador biológico que confirme o excluya el diagnóstico, además tiene una prevalencia estimada de entre el 5-10 \%, e incluso algunas valoraciones han alcanzado el 17 \% (Martínez, 2012).

Teniendo en cuenta la definición consensuada por la Asociación Internacional de Dislexia (Lyon, Shaywitz y Shaywitz, 2003), esta se considera una

dificultad especifica de aprendizaje de origen neurobiológico, caracterizada por la presencia de dificultades en la precisión y fluidez en el reconocimiento de palabras (escritas) y por un déficit en las habilidades de decodificación (lectora) y deletreo. Estas dificultades son normalmente consecuencia de un déficit en el componente fonológico del lenguaje y se presentan de manera inesperada, dado que otras habilidades cognitivas se desarrollan con normalidad y la instrucción lectora es adecuada [la traducción es nuestra].

También se manifiesta en fallas para reconocer de manera precisa y fluida las palabras y en dificultades en ortografía y en decodificación suponiendo consecuencia directa en el desempeño académico de los escolares, pues afecta la comprensión de lectura y la ganancia de vocabulario (Jiménez, 2012; Carrillo y Alegría, 2009), ya que después de comparar el desempeño en diferentes aspectos de la fonología, como el procesamiento del input fonológico, el acceso a las representaciones y el ensamblaje de los fonemas de niños disléxicos con el de normolectores, se corroboran las fallas y el atraso del primer grupo en relación con la práctica en tareas metafonológicas, que podrían indicar la presencia de un trastorno fonológico que entonces explicaría las dificultades que tienen para aprender a leer los niños disléxicos.

No obstante, Leal y Suro (2003) hacen una crítica relacionada con el abordaje diagnóstico de los trastornos de lectura, proponen ampliar y mejorar la hipótesis fonológica, que tiende a generalizarse respecto de la dislexia. Asimismo, plantean la necesidad de ahondar en la evaluación de la conciencia fonológica e interpretar en un rango más amplio sus variables, para procurar un mejor tratamiento no solo terapéutico sino pedagógico. En respuesta a esta orientación, Fostick et al. (2014) estudiaron la probable relación causal entre el procesamiento auditivo y la conciencia fonológica, en un sentido más profundo, comparando lectores normales con lectores disléxicos, tras lo cual concluyeron que el entrenamiento auditivo surte efecto positivo en las tareas relacionadas con la conciencia fonológica y que están directamente relacionadas. Además se tiene en cuenta que la caracterización diagnóstica de la dislexia no es estática y puede afectar diversos aspectos del procesamiento cognitivo-lingüístico y no solo uno, como tradicionalmente se pensaba.

También es importante considerar las características ortográficas de los sistemas alfabéticos y su relación con la dislexia, dado que varían según la transparencia u opacidad de la lengua. Según Seymour, Aro y Erskine (2003), en niños de habla inglesa, las habilidades básicas de decodificación, como las relacionadas con la complejidad silábica y la profundidad ortográfica, pueden desarrollarse con menor suficiencia y afectar tanto la lectura de palabras como la de pseudopalabras, ya que el proceso de adquisición de la lengua escrita es más lento en inglés que en otras lenguas de ortografías similares de Europa. Podría ser que la ortografía en inglés, al ser relativamente irregular, pueda amplificar las dificultades de decodificación de los niños disléxicos, especialmente con respecto a la lectura de no palabras. Dicho planteamiento es considerado también por Matute y Guajardo (2012, pp. 4042) al indicar que la transparencia o la opacidad de un sistema de escritura potencialmente podría ser el elemento lingüístico que más se relaciona con el nivel de dificultad en el aprendizaje de la lectura y puede incidir en la caracterización del trastorno y en su frecuencia de aparición. 
Así pues, las características de una lengua pueden afectar el perfil de la dislexia. En esta medida, la dislexia fonológica se presenta con menos frecuencia en español que en inglés. De hecho, la simplicidad de la estructura del español y la transparencia de su ortografía pueden promover el desarrollo de los procesos fonológicos a una edad más temprana del proceso de aprendizaje de la lectura que la que se aprecia para el inglés (Jiménez y Ramírez, 2002). Sin embargo, Ziegler et al. (2003) estudiaron dicho fenómeno de prevalencia de fallas en el proceso lector en niños de habla inglesa y la compararon con otra lengua europea (alemán). La población presentaba dislexia y fue evaluada con instrumentos similares. Los resultados sugieren que en ambas lenguas se afectan de manera importante las habilidades de decodificación en tareas de lectura de no palabras, velocidad-precisión y el mecanismo fonológico; los niños disléxicos de estos dos países presentaron puntuaciones similares.

Como variable de alto impacto, se encontró que los factores ambientales relacionados con la afectación en el desarrollo adecuado de la lectura (Ardila, Rosselli, Matute y Guajardo, 2005), tales como "el nivel socioeconómico de la familia, el nivel educativo de los padres y los métodos de crianza e instrucción [la traducción es nuestra]", pueden llegar a influir en el desarrollo cognitivo. En este sentido, el desarrollo del lenguaje se puede ver influenciado por variables culturales y determinado por los medios socioeconómicos que pone en desventaja uno frente a otro y repercute en la adquisición de la lectura. Así, los países latinoamericanos presentan una importante dispersión socioeconómica. Por ejemplo, en

un estudio llevado a cabo en México en 2002 se encontró que el 8.3 \% de los mexicanos empleados en áreas urbanas contaba con menos de 3 años de educación, el 20.9 \% tenía una formación educativa de 6 años, el 25.8 \% tenía algún tipo de educación secundaria, el 20.43 \% tenía el equivalente a un diploma de secundaria y el $23.7 \%$ alcanzaba un grado universitario [la traducción es nuestra]. (World Bank, 2005)
Esta situación definitivamente redunda negativamente en procesos de lectura y escritura. Tal efecto también se ha medido a través de la comparación del desarrollo de habilidades metalingüísticas y el aprendizaje lector. Según Aravena (2014), en su estudio comparativo del desempeño de dichas habilidades entre escolares de primer grado de colegios tanto públicos como privados, los resultados sugieren la importancia que tiene el nivel socioeconómico y el tipo de colegio. En este sentido, los niños de los colegios privados mostraron mejor desempeño en las pruebas y corroboraron la gran influencia de entorno en la adquisición de la lectura.

Por otro lado, atender tempranamente desde la escuela las tareas relacionadas con conciencia fonológica actúa como predictor importante del buen desempeño en labores de lectura, ortografía y rendimiento matemático, y puede contrarrestar en alguna medida lo ya descrito. Según Krajewski y Schneider (2009), la evaluación temprana y el abordaje en la escuela de la conciencia fonológica se relacionan directamente con el desarrollo de la alfabetización y otras competencias.

Como ya se mencionó, el trastorno de lectura se ha asociado al déficit fonológico; sin embargo, en los casos de comorbilidad con otras dificultades, como el trastorno por déficit de atención e hiperactividad, resulta difícil ajustarse a tal convención. Aun así, la investigación ha logrado aclarar la influencia fonológica e incluso léxica sobre la conciencia fonológica en niños con dislexia u otras alteraciones, como el trastorno específico del lenguaje. Según Farquharson et al. (2014), en la población con estas características, se logró determinar que, en ambos casos, dislexia y trastorno específico del lenguaje, se aprecia un déficit importante o inmadurez en tareas referidas a la conciencia fonológica y lexical (eliminación de fonemas, aprendizaje y repetición de palabras).

Dos teorías parecen responder a dichas influencias: por un lado, las dificultades para almace- 
nar y procesar los fonemas de una palabra; y por el otro, las implicadas en los rasgos léxicos. Es importante anotar que, en los niños disléxicos, se asume que hay déficit de procesamiento fonológico. Adicionales son los hallazgos de dificultad léxica que influyen en la conciencia fonológica y la necesidad de una detección y un diagnóstico diferencial, con miras a optimizar las estrategias de intervención.

El abordaje pedagógico de la conciencia fonológica es fundamental, de esta manera el éxito en la adquisición de la lectura inicia en la escuela (Bravo, 2005), dado que es allí donde se potencian las habilidades individuales de los niños y las niñas que se combinan con una metodología específica estimuladora de la progresión cognitiva. En cuanto a esta labor, las estrategias son variadas e incluyen el entrenamiento y la relación directa que se cree entre la lectura y el lenguaje oral. En muy importante que el docente pueda detectar cualquier dificultad en la representación oral del lenguaje de los preescolares y llegar a distinguir otros signos de alarma, como los déficits en el desarrollo auditivo, ya que se pueden relacionar directa o causalmente con la dislexia. En el caso terapéutico, el diagnóstico y abordaje a menudo le corresponden al terapeuta del lenguaje. Un ejemplo de ello es demostrado por Zayed et al. (2013), en el que el fonoaudiólogo es el encargado de la aplicación de pruebas (previa autorización en todas las instancias). En este caso específico, con una serie de tareas fonológicas (discriminación fonológica, articulación, identificación de rimas, sonido inicial y reconocimiento fonema-grafema), la conclusión determinó que los niños en riesgo de presentar dislexia mostraban dificultades acentuadas en la identificación de rimas; en otros casos, el abordaje terapéutico de la conciencia fonológica ha sido estudiado a la luz de las implicaciones reales del trabajo sobre esta área comparando los efectos de la intervención en niños con trastornos fonológicos y niños con normodesarrollo del habla y del lenguaje que denota una mejoría en este aspecto. Sin embargo, cuando hay una patología de base como en niños con párkinson, es importante considerar las variables y ser más prudentes en dichas afirmaciones (Harrison, 2008).

Los trabajos disciplinares particulares son muy valiosos, sin embargo, la labor interdisciplinar es fundamental, tal y como lo explican Alvarado et al. (2007), quiene exponen la necesidad de coordinar esfuerzos entre la familia, los profesionales externos y el centro educativo, para conseguir los mejores resultados de intervención en el caso de los alumnos con dislexia. La escuela puede contribuir a través de adaptaciones curriculares individualizadas no significativas y de aula, que contengan estimulación multisensorial, e incluir a estos niños y garantizar el éxito escolar, o puede implementar programas de estimulación de las habilidades de conciencia fonológica en el aula (Arancibia et al., 2012), pues se ha comprobado la eficacia de este tipo de estrategias en el aumento de las habilidades metafonológicas de los preescolares, que los prepara para el inicio lector, más teniendo en cuenta que la investigación aporta datos concluyentes al respecto. De acuerdo con Márquez y De la Osa (2003, p. 357), hay evidencia clara de la "relación lineal significativa entre las tareas de conciencia fonológica y decodificación lectora". Así pues, es fundamental atender la necesidad de detectar los signos predictivos de dificultades lectoras para prevenir consecuencias en el aprendizaje.

En conclusión, la posibilidad de atender desde los primeros años de la escuela las tareas relacionadas con conciencia fonológica ayuda en la detección e incluso en la predicción en un rango importante del desempeño futuro en labores de lectura, ortografía y rendimiento matemático (Krajewski y Schneider, 2009). Además, teniendo en cuenta que tal capacidad puede y debe ser entendida en un marco más amplio, es decir, como una comprensión conceptual y una habilidad que desde el proceso de enseñanza-aprendizaje representa un espacio significativo de instrucción explicita, requiere que el profesor identifique y categorice el nivel de desarrollo de conciencia fonológica de sus estudiantes y 
así pueda brindar las estrategias necesarias de estimulación que permitan transferir habilidades en relación con la lectura como proceso propiamente dicho, o en el caso de que su estudiante requiera un énfasis en alguna tarea e incluso necesite orientar la intervención de otra manera (Taylor, 2008). En este sentido, también es sustancial considerar la exposición e instrucción en una segunda lengua, que, aunque depende de múltiples factores, respecto de la conciencia fonológica, la evidencia sugiere que tiene una construcción única a través de los idiomas.

Por otro lado, la posibilidad de identificar, evaluar y diagnosticar desde el contexto terapéutico fonoaudiológico (Cervera-Mérida e Ygual-Fernández, 2003) permite el abordaje de las habilidades fono- lógicas de una manera puntual que reconoce y diferencia entre dificultades para el reconocimiento de fonemas, relacionadas con el procesamiento del habla o las que se presentan aisladamente, en patologías de habla y lenguaje o en trastornos de lenguaje asociados. Además, la experticia de esta disciplina permite perfilar las características individuales y encauzar las acciones terapéuticas de la mejor manera, pero también facilitar la intervención integradora entre casa, colegio y el espacio clínico. Según Gillon y McNeill (2007), el trabajo oportuno e integrador redunda en mayor éxito en la adquisición de la lectura, a través de estrategias como programas dirigidos a preescolares y escolares, los cuales han indicado eficacia en el rendimiento en labores de conciencia fonológica.

\section{Referencias}

Aguilar Villagrán, M., Marchena Consejero, E., Navarro Guzmán, J. I., Menacho Jiménez, I. y Alcalde Cuevas, C. (2011). Niveles de dificultad de la conciencia fonológica y aprendizaje lector. Revista de Logopedia, Foniatría y Audiología, 31(2), 96-105.

Alvarado, H., Damians, M. A., Gómez, E., Martorell, N., Salas, A.y Sancho, S. (2007). Dislexia, detección, diagnóstico e intervención interdisciplinar. Revista ENGINY, 16-17, 1-26.

Anthony, J. L. y Francis, D. J. (2005). Development of phonological awareness. Current Directions in Psychological Science, 14(5), 255-259.

Arancibia, B., Bizama, M. y Sáez, K. (2012). Aplicación de un programa de estimulación de la conciencia fonológica en preescolares de nivel transición 2 y alumnos de primer año básico pertenecientes a escuelas vulnerables de la Provincia de Concepción, Chile. Revista Signos, 45(80), 236-256.

Aravena, J. (2014). Conciencia fonológica en escolares de primero básico. Revista Chilena de Fonoaudiología, 13, 40-49.

Ardila, A.y Ostrosky, F. (2012). Guía para el diagnóstico neuropsicológico. Florida: American Board of Professional Neuropsy chology.

Ardila, A., Rosselli, M., Matute, E.y Guajardo, S. (2005). The influence of the parents' educational level on the development of executive functions. Developmental Neuropsychology, 28(1), 539-560. 
Artigas-Pallarés, J. (2009). Dislexia: enfermedad, trastorno o algo distinto. Revista de Neurología, 48(2), 63-69.

Baddeley, A. (2003). Working memory: Looking back and looking forward. Nature Reviews Neuroscience, 4(10), 829-839.

Baddeley, A. (2012). Working memory: Theories, models, and controversies. Annual Review of Psychology, 63, 1-29.

Baddeley, A. D., Kopelman, M. D.y Wilson, B. A. (eds.) (2002). The handbook of memory disorders (2. ${ }^{\text {a }}$ ed.). Hoboken, Nueva Jersey: John Wiley \& Sons.

Bizama, M., Arancibia, B. y Sáez, K. (2013). Intervención psicopedagógica temprana en conciencia fonológica como proceso metalingüístico a la base de la lectura en niños de 5 a 6 años socialmente vulnerables. Estudios Pedagógicos, 39(2), 25-39.

Branum-Martin, L., Tao, S. y Garnaat, S. (2014). Bilingual phonological awareness: Reexamining the evidence for relations within and across languages. Journal of Educational Psychology, 107(1), 111-125. http://dx.doi. org/10.1037/a0037149

Bravo Valdivieso, L. (2002). La conciencia fonológica como una zona de desarrollo próximo para el aprendizaje inicial de la lectura. Estudios Pedagógicos, 28, 165-177.

Bravo Valdivieso, L. (2005). Lenguaje y dislexias: enfoque cognitivo del retardo lector. Santiago de Chile: Ediciones Universidad Católica de Chile.

Bravo Valdivieso, L. (2006). Lectura inicial y psicología cognitiva (2. ${ }^{a}$ ed.). Santiago de Chile: Ediciones Universidad Católica de Chile.

Carrillo Gallego, M. y Alegría Isoca, J. (2009). Exploración de las habilidades fonológicas en escolares disléxicos: teoría y práctica. Revista de Logopedia, Foniatría y Audiología, 29(2), 115-130.

Cervera-Mérida, J. F. e Ygual-Fernández, A. (2003). Intervención logopédica en los trastornos fonológicos desde el paradigma psicolingüístico del procesamiento del habla. Revista de Neurología, 36(1), 39-53.

Coloma Tirapegui, C. J., Cárdenas Gajardo, L. R. y De Barbieri Ortiz, Z. (2005). Conciencia fonológica y lengua escrita en niños com trastorno específico del lenguaje expresivo. Revista Cefac, 7(4), 419-25.

Coll Andreu, M. (2011). Plasticidad cerebral y experiencia:fundamentos neurobiológicos de la educación. Ponencia presentada en XII Congreso Internacional de Teoría de la Educación, Universitat Autònoma de Barcelona, Barcelona.

Cuadro, A. y Trías, D. (2008). Desarrollo de la conciencia fonémica: evaluación de un programa de intervención. Revista Argentina de Neuropsicología, 11, 1-8.

Cuetos Vega, F. (2011). Psicología de la lectura: diagnóstico y tratamiento. Madrid:Wolters Kluwer. 
ISSN 0123-1294 | e-ISSN 2027-5358 | Educ.Educ. Vol. 20. No. 2 | Mayo-agosto de 2017 | pp. 175-190.

Universidad de La Sabana | Facultad de Educación

Éva, G. y Réka, O. (2013). The relationship between the first language phonological awareness and the second language reading ability and the cross-language transfer of the first language phonological skills. Transylvanian Journal of Psychology, 14(2), 173-193.

Farquharson, K., Centanni, T. M., Franzluebbers, C. E. y Hogan, T. P. (2013). Phonological and lexical influences on phonological awareness in children with specific language impairment and dyslexia. Frontiers in Psychology, 5, 838-838. doi: 10.3389/fpsyg.2014.00838

Fostick, L., Eshcoly, R., Shtibelman, H., Nehemia, R. y Levi, H. (2014). Efficacy of temporal processing training to improve phonological awareness among dyslexic and normal reading students. Journal of Experimental Psychology: Human Perception and Performance, 40(5), 1799.

Gillon, G. y McNeill, B. (2007). An integrated phonological awareness programme for preschool children with speech disorder. Christchurch: College of Education.

Gómez, L., Duarte, A. M., Merchán, V., Aguirre, D. y Pineda, D. (2007). Conciencia fonológica y comportamiento verbal en niños con dificultades de aprendizaje. Universitas Psychologica, 6(3), 571-580.

Harrison, R. H. (2008). Critical review: The effect of phonological awareness intervention on the speech. Recuperado de https://www.uwo.ca/fhs/lwm/ebp/reviews/2007-08/Harrison,RH.pdf

Herrera, L. y Defior, S. (2005). Una aproximación al procesamiento fonológico de los niños prelectores: conciencia fonológica, memoria verbal a corto plazo y denominación. Psykhe, 14(2), 81-95.

Jamet, E. (2006). Lectura y éxito escolar. Buenos Aires: Fondo de Cultura Económica.

Jiménez González, J. E. (coord.) (2012). Dislexia en español: prevalencia e indicadores cognitivos, culturales, familiares y biológicos. Madrid: Pirámide.

Jiménez González, J. E. y Ramírez Santana, G. (2002). Identifying subtypes of reading disability in the Spanish language. The Spanish Journal of Psychology, 5(1), 3-19.

Krajewski, K.y Schneider,W. (2009). Exploring the impact of phonological awareness, visual-spatial working memory, and preschool quantity-number competencies on mathematics achievement in elementary school: Findings from a 3-year longitudinal study. Journal of Experimental Child Psychology, 103(4), 516-531.

Leal, F.y Suro, J. (2003). La hipótesis fonológica de la dislexia: una crítica constructiva. En M. A. Peredo, Diez estudios sobre la lectura (pp. 265-287). Guadalajara: Editorial Universitaria.

Lyon, G. R., Shaywitz, S. E. y Shaywitz, B. A. (2003). A definition of dyslexia. Annals of Dyslexia, 53(1), 1-14.

Márquez, J. y De la Osa Fuentes, P. (2003). Evaluación de la conciencia fonológica en el incio lector. Anuario de Psicología, 34(3), 357-370.

Martínez, F. (2012). El reto de la dislexia: entender y afrontar las dificultades de aprendizaje. Barcelona: Plataforma Actual. 
Matute, E. y Guajardo, S. (2012). Dislexia: definición e intervención en hispanohablantes (2. ${ }^{a}$ ed.). México: Manual Moderno.

Michalczyk, K., Krajewski, K., Preßler, A. L. y Hasselhorn, M. (2013). The relationships between quantity-number competencies, working memory, and phonological awareness in 5-and 6-year-olds. British Journal of Developmental Psychology, 37(4), 408-424.

Ministerio de Educación, Cultura y Deporte Español (2012). La atención al alumnado con dislexia en el sistema educativo en el contexto de las necesidades específicas de apoyo educativo. Recuperado de http://www. asandis.org/doc/la-atencion-al-alumnado-con-dislexia_MECD.pdf

Núñez Delgado, M. P.y Santamarina Sancho, M. (2014). Prerrequisitos para el proceso de aprendizaje de la lectura y la escritura: conciencia fonológica y destrezas orales de la lengua. Lengua y Habla, 18, 72-92.

Preston, J. L., Hull, M.y Edwards, M. L. (2013). Preschool speech error patterns predict articulation and phonological awareness outcomes in children with histories of speech sound disorders. American Journal of SpeechLanguage Pathology, 22(2), 173-184.

Rachmani, R. M. (2011). The effects of a phonological awareness and alphabet knowledge intervention on four year old kindergarten children (Tesis de maestría, University of Waikato, Hamilton, Nueva Zelanda).

Rosselli, M. y Matute, E. (2012). Importancia de los factores lingüísticos y ambientales en el diagnóstico de Dislexia. En E. Matute y S. Guajardo, Dislexia: definición e intervención en hispanohablantes (2. ${ }^{a}$ ed., pp. 3-14). México: Manual Moderno.

Rosselli, M., Matute, E.y Ardila, A. (2010). Neuropsicología del desarrollo infantil. Bogotá: Manual Moderno.

Seymour, P. H., Aro, M. y Erskine, J. M. (2003). Foundation literacy acquisition in European orthographies. British Journal of Psychology, 94(2), 143-174.

Solsona, J., Navarro, J. I. y Aguilar, M. (2005). Conocimiento lógicomatemático y conciencia fonológica en educación infantil. Revista de Educación, 341, 781-801.

Su, I. F., Lau, D. K., Zhang, Z., Yan, N. y Law, S. P. (2011). Deficits in processing characters in chinese developmental dyslexia: Preliminary results from event-related potentials and time frequency analyses. International Journal of Linguistics, 3(1), 1-13.

Taylor, B. (2008). What does excellent reading instruction. Minnesota: Minnesota Center for Reading Research.

Tirapu-Ustárroz, J. y Muñoz-Céspedes, J. (2005). Memoria y funciones ejecutivas. Revista de Neurología, 41(8), 475-484.

Vargas, A. y Villamil, W. (2007). El papel de la conciencia fonológica como habilidad subyacente al alfabetismo temprano y su relación en la comprensión de la lectura y la producción escrita de textos. Pensamiento Psicológico, 3(9), 163-174. 
ISSN 0123-1294 | e-ISSN 2027-5358 | Educ.Educ. Vol. 20. No. 2 | Mayo-agosto de 2017 | pp. 175-190.

Universidad de La Sabana | Facultad de Educación

World Bank (2005). Summary education profile for Mexico. Recuperado de http://devdata.worldbank.org/edstats/SummaryEducationProfiles/CountryData/GetShowData.asp?sCtry=MEX,Mexico

Zayed, A. M., Roehrig, A., Arrastia-Lloyd, M. C.y Gilgil, N. M. (2013). Phonological Awareness and working memory in Arabic-speaking Egyptian preschool Children at Risk for Dyslexia. International Journal of Psychological Studies, 5(1), 139-149.

Ziegler, J. C., Perry, C., Ma-Wyatt, A., Ladner, D. y Schulte-Körne, G. (2003). Developmental dyslexia in different languages: Language-specific or universal? Journal of Experimental Child Psychology, 86(3), 169-193. 